\title{
Simultaneous Progression and Regression of Coronary Artery Disease in a Patient from North India
}

\author{
Arora $\mathrm{H}^{1 *}$, Parekh $\mathrm{S}^{1}$ and Mohanty $\mathrm{A}^{2}$ \\ ${ }^{1}$ Consultant Cardiologist, Grecian Super Speciality Hospital, India \\ ${ }^{2}$ Senior Consultant Cardiologist, Sir Gangaram Hospital, India
}

\section{Case Report}

Volume 2 Issue 4

Received Date: August 09, 2018

Published Date: September 03, 2018

*Corresponding author: Dr. Himanshu Arora, Consultant Cardiologist, Grecian Super Speciality Hospital, Mohali, India, Tel: +91-9818742632; Email: himanshu3105shs@gmail.com

\section{Abstract}

Background: Lipids have a central role in the pathogenesis of atheroma formation. Statins are known to favorably alter the morphology of plaque and decrease the atheroma volume.

Case: We present a case of 45 year old male who had spontaneous regression of coronary artery disease in Left anterior descending (LAD) with progression of coronary artery disease in left circumflex (LCX) and right coronary artery (RCA) over a period of 7 years.

Conclusion: Disease regression could be attributable to statins while mechanism for simultaneous progression in other two vessels is still unclear.

Keywords: Statins; Progression; Regression; Coronary Artery Disease

\section{Case Presentation}

45 year old male, known case of old anterior Non-ST elevation myocardial infarction (2009), presented with complaints of shortness of breath \& chest heaviness. His pulse was $82 / \mathrm{min}$, blood pressure was $130 / 70 \mathrm{~mm} \mathrm{Hg}$, no cyanosis or pedal edema, JVP was not raised. Systemic examination was normal.

Patient had undergone coronary angiography (CAG) in 2009 which revealed mid LAD 70\% eccentric stenosis and RCA showed mild plaquing, remaining coronaries were normal (Figure 1). Patient was then advised for percutaneous coronary intervention (PCI) to LAD, but he was not willing for the same, hence he was discharged on optimal medical treatment. His treatment consisted of Aspirin 75mg, Clopidogrel 75mg, Atorvastatin 40mg, Ezetimibe $10 \mathrm{mg}$, Ramipril $2.5 \mathrm{mg} \quad \& \quad$ Isosorbide mononitrate $30 \mathrm{mg}$ daily.

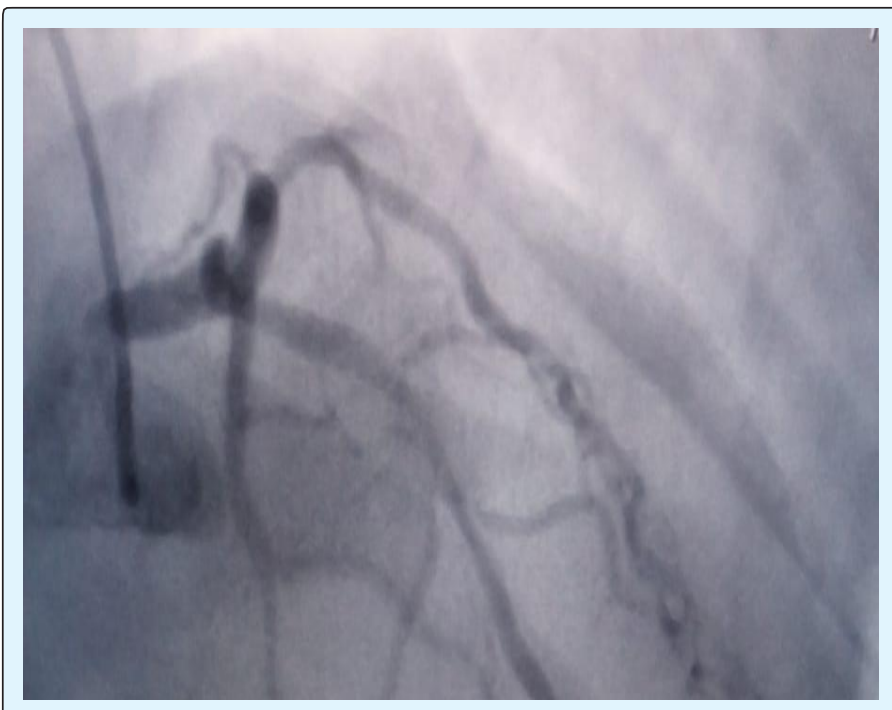

Figure 1: Coronary angiogram showing LAD mid segment $70 \%$ stenosis. 


\section{Open Access Journal of Cardiology}

Patient continued the treatment with good compliance and was on regular follow up in outpatient department. However he was admitted again as a case of Acute Coronary Syndrome in 2016. His Echo showed regional wall motion abnormality, mild MR, trace pericardial effusion and ejection fraction of $40 \%$. He underwent coronary angiography which revealed left main normal, LAD 20\% residual stenosis (Figure 2), LCX proximal \& mid $90 \%$ tandem lesions, RCA proximal plaquing followed by mid to distal diffuse $95 \%$ lesion with thrombus \& spontaneous spiral dissection (Figure 3), Ramus ostial plaquing.

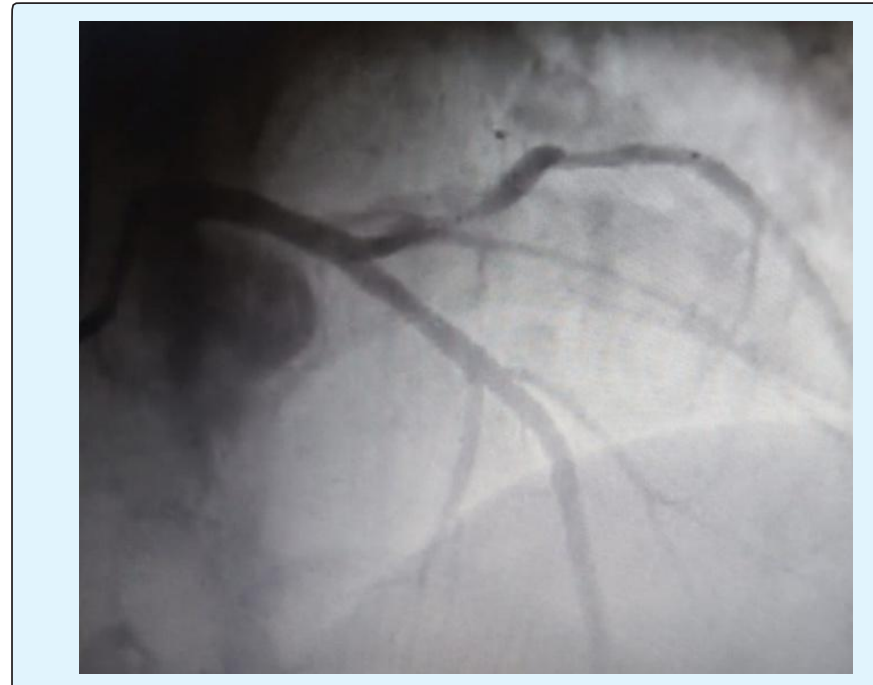

Figure 2: Coronary angiogram showing regression of LAD lesion with residual $20 \%$ stenosis.

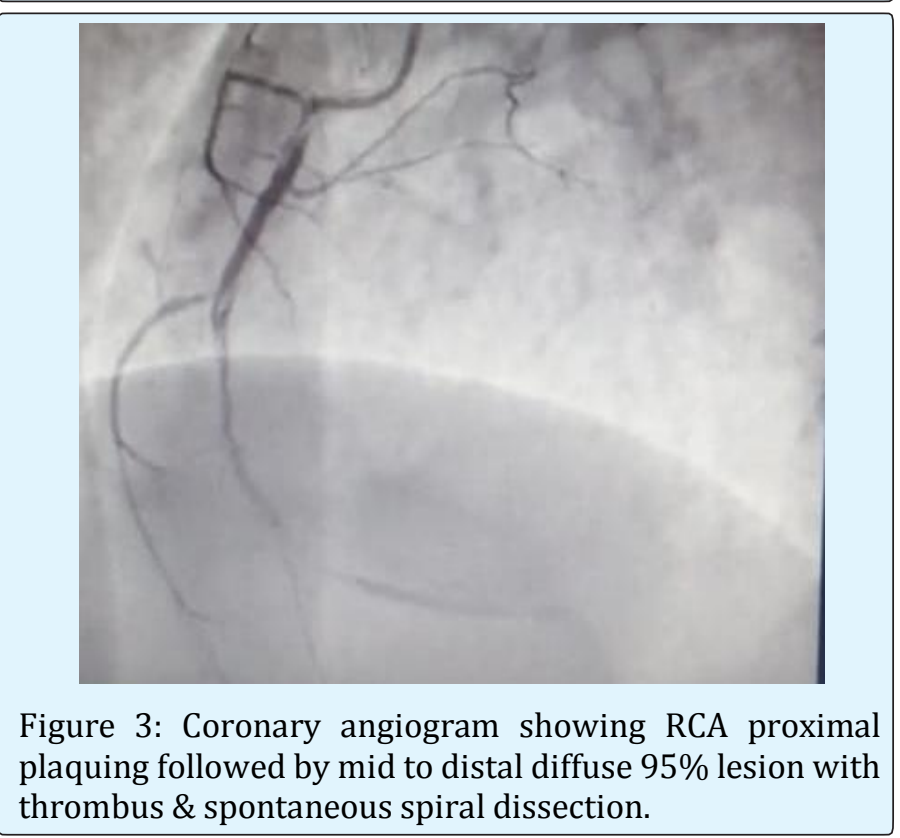

CAG was consistent with regression of CAD in LAD \& progression of CAD in RCA \& LCx.

Subsequently he underwent PCI and stenting to RCA (3 stents) and LCx (1 stent) using everolimus eluting stents with good end results.

\section{Discussion}

Plaque reversal occurs by removal of lipids and necrotic material, endothelial repair, or halt of vascular smooth muscle cell proliferation. Serial CTA evaluation of coronary plaques allows for the assessment of interval change in the plaque morphology. Statin treatment results in decrease in the plaque and necrotic core volume [1]. Statin treatment induces favorable plaque morphologic changes with an increase in fibrous cap thickness, and decreases in both percentage plaque and lipid volume indexes [2].

In a meta-analysis of eight trials, 919 patients (461 patients in the statin group and 458 in the placebo group) were studied with intravascular ultrasound (IVUS). There was no significant difference between the two groups in terms of their plaque characteristics at baseline. However, there was a statistically significant mean difference in coronary atheroma volume between the statin therapy and the placebo arms, which was $-3.573(\mathrm{P}<0.01)$. This suggests that statins have the potential to induce plaque reversal [3].

One small study analyzed the effects of rosuvastatin combined with ramipril on atheroma volume and its mechanism in patients with intermediate CAD. In this study, 21 patients received rosuvastatin (20mg daily) and 19 patients received rosuvastatin along with ramipril (20mg and $10 \mathrm{mg}$, respectively) for 9 to 12 months. There was decrease in the TAV in both groups, with reduction in the CRP levels in the combination treatment group [4].

Hibi, et al. studied effects of statin treatment on plaque regression in patients with polyvascular disease versus those with CAD alone. They studied 252 patients (at 33 centers) with a history of an ACS, who underwent percutaneous intervention to localize the lesion followed by treatment with atorvastatin (20mg per day) or pitavastatin (4mg per day). Both groups showed regression of plaques, as assessed by IVUS at baseline and at 8-12 months follow-up [5].

In a prospective randomized comparative study using rosuvastatin $20 \mathrm{mg}(\mathrm{n}=65)$ and atorvastatin $40 \mathrm{mg}(\mathrm{n}=$ 


\section{Open Access Journal of Cardiology}

63), IVUS was used at baseline and at 11-month followup, to show effective plaque regression. TAV and percent atheroma volume (PAV) was measured. Plaque was decreased in 99 of 128 patients (77\%); (85\% in the rosuvastatin group vs $70 \%$ in the atorvastatin group).

Both groups showed change in TAV: $-4.4 \pm 7.3 \mathrm{~mm}^{3}$ for the rosuvastatin group and $-3.68 \pm 6.8 \mathrm{~mm}^{3}$ for the atorvastatin group $(\mathrm{P}=0.5)$. The difference in PAV between the two groups was not statistically significant $(P=0.14)$. These results demonstrate that both statins are effective in reducing plaque burden [6].

Kovarnik, et al. randomized 89 patients to receive either atorvastatin $80 \mathrm{mg}$ plus ezetimibe $10 \mathrm{mg}$ or standard treatment per the patients' general practitioner for 12 months. The authors found a decrease in the coronary artery PAV $(-0.4 \%)$ in the group on combination treatment versus an increase $(+1.4 \%)$ in the other group $(P=0.014)$, as measured by IVUS. There was also an increased frequency of combined atherosclerosis regression (increased lumen volume plus decreased PAV) in patients taking both medications $(40.5 \%)$ compared with the group on monotherapy (14.9\%) $(\mathrm{P}=0.007)$ [7].

The literature available suggests that statins play an important role in plaque regression. However in our case it was surprising to have disease regressed in one vessel while it progressed in the other two vessels. The exact mechanism is still debatable.

\section{Conclusion}

Major reductions in coronary events are associated with aggressive medical therapy. Statin therapy is associated with beneficial histological changes in plaque morphology as demonstrated by IVUS. The cellular and molecular mechanisms responsible for plaque regression in humans are less understood. Our case represents a unique combination of simultaneous regression of disease in LAD with progression in LCX and RCA, despite giving statin therapy. More studies are needed to clearly elucidate this issue.

\section{References}

1. Inoue K, Motoyama S, Sarai M, Sato T, Harigaya H, et al. (2010) Serial coronary CT angiography-verified changes in plaque characteristics as an end point. JACC Cardiovasc Imaging 3(7): 691-698.

2. Hattori $\mathrm{K}$, Ozaki $\mathrm{Y}$, Ismail TF, Okumura M, Naruse $\mathrm{H}$, et al. (2012) Impact of statin therapy on plaque characteristics as assessed by serial OCT, grayscale and integrated backscatter-IVUS. JACC Cardiovasc Imaging 5(2): 169-177.

3. Bedi U, Singh M, Singh P, Molnar J, Khosla S, et al. (2011) Effects of statins on progression of coronary artery disease as measured by intravascular ultrasound. J Clin Hypertens (Greenwich) 13(7): 492496.

4. Han SH, Chung WJ, Kang WC, Lee K, Park YM, et al. (2012) Rosuvastatin combined with ramipril significantly reduced atheroma volume by antiinflammatory mechanism: Comparative analysis with rosuvastatin alone by intravascular ultrasound. Int J Cardiol 158(2): 217-224.

5. Hibi K, Kimura T, Kimura K, Morimoto T, Hiro T, et al. (2011) Clinically evident polyvascular disease and regression of coronary atherosclerosis after intensive statin therapy in patients with acute coronary syndrome: serial intravascular ultrasound from the Japanese assessment of pitavastatin and atorvastatin in acute coronary syndrome (JAPAN-ACS) trial. Atherosclerosis 219(2): 743-749.

6. Hong YJ, Jeong MH, Hachinohe D, Ahmed K, Choi YH, et al. (2011) Comparison of Effects of rosuvastatin and atorvastatin on plaque regression in Korean patients with untreated intermediate coronary stenosis. Circ J 75(2): 398-406.

7. Kovarnik T, Mintz GS, Skalicka H, Kral A, Horak J, et al. (2012) Virtual histology evaluation of atherosclerosis regression during atorvastatin and ezetimibe administration: HEAVEN study. Circ J 76(1): 176-183.

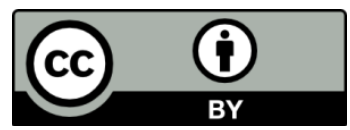

Arora H. Simultaneous Progression and Regression of Coronary Artery Disease in a Patient from North India. J Cardiol 2018, 2(4): 000130. 